\title{
An Anthropological Approach to an Occupational Intervention: The Exercise and Dementia Project
}

\author{
Margaret A. Perkinson, PhD \\ Department of Occupational Science \& Occupational Therapy \\ Doisy College of Health Sciences \\ Saint Louis University
}

The growing attention of occupational science/ occupational therapy to issues of social inequality, social marginalization, and representation of diverse voices, as seen in recent publications such as A Political Practice of Occupational Therapy (Pollard, Sakellariou, \& Kronenberg 2009) and Occupational Therapy without Borders (Kronenberg, Algado, \& Pollard 2005), comes at a time when anthropology also is re-considering its central roles and moral duties as a discipline. A selective integration of these disciplinary approaches may synergize efforts to address the occupational needs of marginalized persons. This paper describes an interdisciplinary attempt to work with one marginalized group, persons with Alzheimer's disease and their family caregivers, by encouraging the inclusion of physical activity into the daily routines of persons with dementia and enhancing the occupational role of family caregivers.

The shift from a purely biomedical perspective of dementia to one that takes into account the potential for personhood of one with the disease (Kitwood 1997) is a critical step toward the de-marginalization of individuals with Alzheimer's. Using ethnographic methods to examine life experiences of persons with dementia, anthropologists have served a pivotal role in this shift in perspectives (Leibing \& Cohen 2006; McLean 2007). Their work has encouraged a comparable shift in models of dementia care, from simple custodial care to an emphasis on the physical and psychosocial factors impacting quality of life (Perkinson 2000).

The potential for the experience of personhood, improved quality of life, and engagement in meaningful occupations by persons with dementia were basic assumptions that guided the development of the Exercise and Dementia Project. This project employed an anthropological perspective to develop and evaluate an occupational intervention: a family-supervised, home-based physical activity program for persons with mild dementia (Perkinson 2008). Based on a multi-dimensional set of baseline assessments, an interdisciplinary team (occupational therapist, physical therapist, and medical anthropologist) developed and evaluated individualized physical activity programs for 30 persons with mild dementia and their primary family caregivers. Family exercise supervisors received instructions on the performance and safe supervision of the exercise program and exercised with their relatives three times a week for three months. Follow-up assessments revealed significant improvement on various physical measures and general satisfaction with the program.

In addition to collecting traditional, standardized assessments of outcomes, the project evaluation documented and analyzed the processes through which the interventions morphed into personal routines and habits. Ethnographic observations, exercise diaries, in-depth follow-up interviews, and focus groups with family exercise supervisors indicated how participants learned and performed the exercise programs within the context their everyday lives. Data analyses revealed unanticipated physical and social barriers and incentives to performance of and adherence to the intervention. When faced with such barriers, many exercise supervisors adapted by making ad hoc modifications to the exercise programs. They occasionally introduced technical devices to enhance the impact of the program. Family supervisors also developed idiosyncratic criteria to assess the adequacy of exercise performance. The meanings that participants ascribed to their experiences of physical activity and to the occupation of exercise supervision were pivotal to adherence and general impact of the intervention.

Anthropological process evaluation research (Butler 2005), with its focus on context, meaning, and process of implementation can provide insight into the manner in which activities of an intervention are integrated into daily occupations and can reveal the meanings and value of OT interventions from the perspectives of its participants. Productive partnership between anthropology and occupational science/occupational therapy holds promise for the development of an evidence-based field of practice and body of knowledge that will address issues such as social and occupational justice, which are central concerns for both fields. 
Perkinson, continued from page 28

\section{Acknowledgements:}

I wish to acknowledge the support of the national Alzheimer's Association (GMS-4250) for funding this project; the Alzheimer's Association St. Louis Chapter and Washington University School of Medicine Alzheimer's Disease Research Center for their assistance in recruitment; David Sinacore, PT, PhD, for the generous use of his lab for conducting assessments; and the generous and dedicated involvement of the project participants.

\section{References:}

Butler, M.O. 2005. Translating evaluation anthropology. In M.O. Butler \& J. Copeland-Carson (eds.) Creating evaluation anthropology. NAPA Bulletin 24. Berkeley, CA: University of California Press.

Kitwood, T. 1997. Dementia reconsidered: The person comes first. Buckingham, UK: Open University Press.

Kronenberg, F., Algado, S. \& Pollard, N. 2005. Occupational therapy without borders. Edinburgh: Elsevier/Churchill Livingstone.

Leibing, A. \& Cohen, L. (Eds.) 2006. Thinking about dementia: Culture, loss, and the anthropology of senility. New Brunswick, NJ: Rutgers University Press.

McLean, A. 2007. The person in dementia: A study of nursing home care in the U.S. Toronto: Broadview Press.

Perkinson, MA. Family and nursing home staff perceptions of quality of life in dementia. In R. Rubinstein, M. Moss, and M. Kleban (Eds.) The many dimensions of aging. New York, New York: Springer Publishing Company, 2000.

Perkinson, M.A. 2008. Negotiating disciplines: Developing a dementia exercise program. Practicing Anthropology (Special issue on Anthropology and Occupational Therapy), 30(3), 10-15.

Pollard, N., Sakellariou, D. \& Kronenberg, F. 2009. A political practice of occupational therapy. Edinburgh: Elsevier/ Churchill Livingstone.

Briller, continued from page 24

Aging Quarterly has been the first venue in which they authored work and it was a pleasure to see their pride as they looked at their very first publication here.

Finally, another special event for AAGE in June will be the next AAGE workshop entitled "Aging and the Indigenous Peoples of North America" to be held at the University of Oklahoma from June $5^{\text {th }}-9^{\text {th }}$. It promises to be a very exciting conference and what goes on there will be reported on in the next issue of Anthropology and Aging Quarterly. Thanks again to all who contribute your time to make AAGE activities, such as Anthropology and Aging Quarterly and these special meetings, a reality for our membership. On the subject of membership, now that we have Club Express up and running, please feel free to invite your students, colleagues, and others interested to join AAGE and see what we have for them. It's still a real $21^{\text {st }}$ century bargain!

New Publications, continued from page 37

\section{Widow(er)s}

Holtslander, L.F. \& W.D. Duggleby 2009 The hope experience of older bereaved women who cared for a spouse with terminal cancer. Qualitative Health Research 19(3):388-400.

\section{Work}

Research on Aging 31(1), 2009: Special issue on

"Employment opportunities at older ages." 